\title{
Acromegalic arthropathy in various stages of the disease: an MRI study
}

\author{
K M J A Claessen', A Navas Canete², P W de Bruin², A M Pereira1', M Kloppenburg³, \\ H M Kroon ${ }^{2, *}$ and N R Biermasz ${ }^{1, *}$ \\ Departments of ${ }^{1}$ Endocrinology \& Metabolic Diseases and Center for Endocrine Tumors Leiden, ${ }^{2}$ Radiology, and \\ ${ }^{3}$ Rheumatology, Leiden University Medical Center, Leiden, The Netherlands \\ *(H M Kroon and N R Biermasz share last authorship)
}

Correspondence should be addressed to $\mathrm{K}$ M J A Claessen Email

K.M.J.A.Claessen@lumc.nl

\begin{abstract}
Background: Arthropathy is a prevalent and invalidating complication of acromegaly with a characteristic radiographic phenotype. We aimed to further characterize cartilage and bone abnormalities associated with acromegalic arthropathy using magnetic resonance imaging (MRI).

Methods: Twenty-six patients ( $23 \%$ women, mean age $56.8 \pm 13.4$ years), with active $(n=10)$ and controlled acromegaly $(n=16)$ underwent a 3.0 T MRI of the right knee. Osteophytes, cartilage defects, bone marrow lesions and subchondral cysts were assessed by the Knee Osteoarthritis Scoring System (KOSS) method. Cartilage thickness and cartilage $\mathrm{T} 2$ relaxation times, in which higher values reflect increased water content and/or structural changes, were measured. Twenty-five controls ( $52 \%$ women, mean age: $59.6 \pm 8.0$ years) with primary knee OA were included for comparison.

Results: Both in active and controlled acromegaly, structural OA defects were highly prevalent, with thickest cartilage and highest cartilage $\mathrm{T} 2$ relaxation times in the active patients. When compared to primary OA subjects, patients with acromegaly seem to have less cysts $(12 \%$ vs $48 \%, P=0.001)$ and bone marrow lesions ( $15 \%$ vs $80 \%, P=0.006)$, but comparable prevalence of osteophytosis and cartilage defects. Patients with acromegaly had $31 \%$ thicker total joint cartilage $(P<0.001)$ with higher cartilage T2 relaxation times at all measured sites than primary OA subjects $(P<0.01)$. Conclusions: Patients with active acromegaly have a high prevalence of structural OA abnormalities in combination with thick joint cartilage. In addition, T2 relaxation times of cartilage are high in active patients, indicating unhealthy cartilage with increased water content, which is (partially) reversible by adequate treatment. Patients with acromegaly have a different distribution of structural OA abnormalities visualized by MRI than primary OA subjects, especially of cartilage defects.
\end{abstract}

European Journal of Endocrinology

(2017) 176, 779-790

\section{Introduction}

Acromegaly is a rare chronic endocrine disease, caused by a growth hormone (GH)-producing pituitary adenoma, resulting in elevated GH and insulin-like growth factor-1 (IGF-1) concentrations. Patients with acromegaly have an increased risk to develop secondary osteoarthritis (OA), having a considerable impact on physical functioning and psychological well-being $(1,2)$. In $50-70 \%$ of patients, arthropathy is one of the presenting symptoms at diagnosis. The mechanisms that are involved in the pathophysiology of acromegalic joint disease are not fully elucidated. Recent studies point towards a role for excess GH/IGF- 1 activity both in the initiation and in the progression of acromegalic arthropathy $(3,4,5)$. Based on old studies in mainly active patients, it is hypothesized
() 2017 European Society of Endocrinology Printed in Great Britain
Published by Bioscientifica Ltd. 
that there are two phases in the pathogenesis of acromegalic arthropathy. At the initial stage, elevated GH and (local and circulating) IGF-1 levels induce cartilage hypertrophy and laxity of the periarticular ligaments, which result in an altered geometry of the joints and hypermobility. In this phase, radiographic abnormalities include joint space widening and periarticular soft tissue hypertrophy. This early stage is thought to be at least partially reversible by adequate treatment $(6,7)$. However, when the GH excess persists, the disease acquires features of a degenerative joint disease, resulting in cyst and osteophyte formation with further deterioration of joint architecture by disproportionate proliferation of regenerative fibrocartilage (8). In this late phase, changes become irreversible and may be GH-independent, and acromegaly treatment has only limited effects on joint symptoms (9). On conventional radiographs of patients with active acromegaly, joint disease is characterized by widening of joint spaces and severe osteophytosis (10). In a well-characterized cohort of patients with longterm disease control after currently available treatment, i.e., transsphenoidal pituitary surgery or GH-lowering medication, we recently observed a 4 to 12 -fold increased prevalence of arthropathy, already being present at young age (1). Remarkably, the distribution of structural OA features differs from that in patients with primary OA. Acromegalic arthropathy is predominantly characterized by osteophytosis, frequently in combination with preserved or even widened joint spaces, suggesting that cartilage hypertrophy is maintained despite long-term biochemical disease control (11). However, no imaging studies of this unique phenotype of secondary OA with pathological cartilage hypertrophy have been performed, except for a single study that used ultrasonography $(6,12)$. We have recently reported that several parameters indicating an increased GH/IGF-1 signal were indeed associated with radiographic OA (ROA) and ROA progression in acromegalic patients, i.e. high IGF-1 levels at the time of diagnosis and the presence of the common exon 3 deletion (d3-GHR) GH receptor polymorphism $(3,4,5)$.

To study the unique phenotype of acromegalic arthropathy further, magnetic resonance imaging (MRI) may give additional information to radiographs. MRI directly visualizes cartilage, enabling assessment of cartilage defects, thickness and quality, osteophytes but also other structural abnormalities of subchondral bone such as cysts and bone marrow edema. Cartilage quality can be measured by cartilage $\mathrm{T} 2$ relaxation times, being related to water content and collagen anisotropy, providing information on cartilage biochemical composition (13). A higher T2 value has previously been reported in cartilage of patients with OA compared to healthy controls and its higher T2 values were correlated with the severity of the disease $(14,15)$.

In the present study, we investigated knees of 26 patients with acromegaly by 3.0 Tesla (3.0T) MRI to study structural OA abnormalities, with particular interest in cartilage features. We included both acromegaly patients with active and controlled disease to study the potential relationship between structural OA features and disease activity. Subjects with primary OA were included as controls to differentiate the structural MRI abnormalities that were acromegaly-specific.

\section{Subjects and methods}

\section{Study design and patient selection}

\section{Study design}

In a cross-sectional study design, we performed 3.0T MRI scans of the knee in 26 patients with acromegaly, who were divided into two subgroups: (i) active patients and (ii) patients in remission, by transsphenoidal surgery and/or radiotherapy, or medical treatment. Two patients underwent two knee MRIs at different time points (i.e. one MRI before surgery and one MRI $>6$ months after successful surgery), resulting in 28 available MRIs for analysis. We included control subjects with primary knee OA from the geMstoan study (vide infra, (16)) to evaluate acromegalyspecific differences in structural joint abnormalities and cartilage thickness of the knee. In addition, we included reference values obtained from literature, i.e. controls with mild primary OA (13), to evaluate cartilage biochemical composition by measuring cartilage $\mathrm{T} 2$ relaxation times at different locations in the knee.

\section{Patients}

All consecutive patients with acromegaly, who were referred to the Leiden University Medical Center, were collected in a database. Complementary to a crosssectional and follow-up study evaluating clinical and radiographic arthropathy in long-term biochemically controlled patients, a random subset of patients with controlled acromegaly was invited to undergo an additional knee MRI assessment, inviting both patients who were cured after surgery and patients on medical treatment $(1,4)$. In addition, all consecutive new patients with active acromegaly were approached, to compare 
with controlled patients. Previous knee surgery was not an exclusion criterion.

From the beginning of the treatment for acromegaly, patients were followed strictly on a yearly base. The majority of patients underwent transsphenoidal surgery. If no remission was achieved, adjuvant therapy in the form of radiotherapy (prior to 1985) or somatostatin (SMS) analogs (from 1985 onwards) was given. After 1998, some patients (e.g. based on age, comorbidity, patient's preference) received primary medical treatment with depot formulations of long-acting SMS analogs. Since 2003, treatment-resistant patients were treated with pegvisomant.

In all patients, yearly oral glucose tolerance tests (oGTT) (except in medically treated patients), fasting serum GH and IGF-1 levels were measured to assess the disease activity. Patients were considered to be in remission based on the following criteria: a normal glucose-suppressed serum GH $<1.25$ (RIA assay until 1992) or $0.38 \mu \mathrm{g} / \mathrm{L}$ (immunofluorometric assay (IFMA) from 1992 onwards) and normal IGF-1 levels for age and sex (from 1986 onwards). Random serum GH level of $<1.0 \mu \mathrm{g} / \mathrm{L}$ was used as additional criterion for disease remission, only in the patients treated with SMS analogs $(17,18,19)$. During the treatment with pegvisomant, disease control was defined as normalization of IGF-1 levels $(19,20)$. Patients not meeting these criteria repeatedly were offered additional treatment.

Hypopituitarism was supplemented with levothyroxine, hydrocortisone and testosterone/estrogens based on the current guidelines (21). GH deficiency was not routinely assessed.

The study protocol was approved by the Medical Ethics Committee, and all subjects gave written informed consent.

\section{Controls}

Two control groups were included for comparison with patients with acromegaly.

A. For comparison of the structural OA abnormalities assessed by the Knee Osteoarthritis Scoring System (KOSS) and cartilage thickness measurements, patients with acromegaly were compared to controls from the GEneration of Models, Mechanisms \& Markers for Stratification of OsteoArthritis patients (geMstoan) study. The geMstoan study $(N=62)$ is a longitudinal study among primary OA patients with established symptomatic and radiographic knee OA, aiming on identification of new biomarkers for OA progression (16). To compare with our patients with acromegaly, we selected 26 geMstoan subjects with comparable age. However, as the frequency of females was much higher in controls, matching on sex was not completely possible. For one subject, the MRI was missing, resulting in 25 eligible controls. The geMstoan study is approved by the Medical Ethics Committee, and all patients provided written informed consent.

B. For comparison of cartilage T2 relaxation times, we used a literature reference from Stahl et al. describing 17 controls ( 9 females/8 males) with mild primary OA, as reference values of cartilage $\mathrm{T} 2$ relaxation times were not available in our center (13). In this literature reference, OA was defined as radiographic OA (KellgrenLawrence (KL) 1 or 2) and clinical OA according to the clinical ACR criteria (mean age of $54.0 \pm 10.0$ years; mean BMI $23.6 \pm 7.1 \mathrm{~kg} / \mathrm{m}^{2}$ ). Cartilage T2 relaxation times were measured at the same knee locations as in the acromegalic patients with comparable techniques (vide infra).

\section{Study protocol}

Patients with acromegaly were seen on the outpatient clinic for a single study visit. All patients completed standardized questionnaires on demographic data, medical history and OA signs and symptoms, and the validated WOMAC (Western Ontario and McMaster Universities Osteoarthritis Index) questionnaire on pain, stiffness and functional disability of the lower limb. Conventional knee radiographs were obtained according to a standard protocol (vide infra), and all patients underwent an MRI scan of the right knee. Physical examination of the knee was performed by a single physician (K M J A C), trained in structured joint assessment.

Controls from the geMstoan study underwent an MRI of the knee with symptomatic $\mathrm{OA}$, and conventional knee radiographs were obtained. Self-reported pain was assessed by the Visual Analog Scale (VAS, 0-100) within 2 weeks of MRI acquisition and the WOMAC questionnaire was completed.

\section{Study parameters}

\section{Parameters of acromegalic disease}

Active disease duration was estimated from the start of symptoms and signs to the date of normalization of serum IGF-1 concentration after treatment. We calculated duration of remission from the date of biochemical remission until the start of the current study. Criteria for cure and biochemical controls are described above (see paragraph 'Patients'). In this study, cured and biochemically controlled patients were both referred to as 'in remission'. 
Assays

Serum GH was measured with a sensitive immunofluorometric assay (IFMA) (Wallac, Turku, Finland), specific for the $22 \mathrm{kDa} G H$ protein (detection limit: $0.01 \mu \mathrm{g} / \mathrm{L}$, interassay coefficient of variation $(\mathrm{CV})$ : $1.6-8.4 \%$ of $0.01-15.38 \mu \mathrm{g} / \mathrm{L}$ ) from 1992 onwards. For the conversion of $\mu \mathrm{g} / \mathrm{L}$ to $\mathrm{U} / \mathrm{L}$, multiply by 2.6 . Before 1992 , $\mathrm{GH}$ levels were measured by radioimmunoassay (RIA) (Biolab, Serona, Coissins, Switzerland), detection limit: $0.5 \mathrm{U} / \mathrm{L}$, with an interassay $\mathrm{CV}<5 \%$. For the conversion of $\mu \mathrm{g} / \mathrm{L}$ to $\mathrm{U} / \mathrm{L}$, a multiplication factor of 2 was used.

Serum IGF-1 concentrations (nmol/L) were measured using an immunometric technique on an Immulite 2500 system (Siemens Healthcare Diagnostics). Intra-assay variations at mean plasma levels of 8 and $75 \mathrm{nmol} / \mathrm{L}$ were 5.0 and $7.5 \%$ respectively. IGF-1 levels were represented as SDS based on lambda-mu-sigma smoothed reference curves of 906 subjects $(22,23)$.

\section{Radiographic protocol}

Both in patients with acromegaly and controls from geMstoan study, conventional knee radiographs (posterior-anterior (PA), in weight-bearing/semi-flexed and lateral) were obtained, using the same standardized protocol with a fixed film-focus distance and fixed flexionposition (24). Radiographic examinations were performed by a single experienced radiographer. Radiographs were available for 24 of 28 patients with acromegaly, and in all controls from the geMstoan study.

Radiographic knee OA was assessed according to the KL scale by an experienced musculoskeletal radiologist $(\mathrm{H}$ $\mathrm{M} \mathrm{K}$ ) (25), and was defined as $\mathrm{KL} \geq 2$. The reproducibility, depicted by the intra-class correlation coefficient (ICC), was 0.99 and was based on a randomly selected sample of 34 radiographs (17 right and 17 left knees).

\section{MRI protocol/acquisition}

Although 1.5 Tesla (1.5 T) MRI of the knee is standard in clinical practice and performs well in assessing internal joint derangement, limitations have to be considered. In particular, assessing abnormalities of the hyaline articular cartilage and lateral meniscus may be challenging. The current literature suggests that imaging at 3.0 T MRI offers clinical benefits compared to $1.5 \mathrm{~T}(26,27)$. The stronger field strength of the $3 \mathrm{~T}$ MRI increases signal-to-noise ratio (SNR), potentially allowing thinner sections and higher in plane spatial resolutions resulting in better visualization of anatomical and pathological structures (28). Therefore we scanned our patients on a $3 \mathrm{~T}$ MRI for the best interpretation of the images.

Patients: MRI scans were obtained using a $3.0 \mathrm{~T}$ superconducting MRI scanner (Gyroscan Achieva; Philips Medical Systems, Best, the Netherlands) and an eight-channel knee coil, and were performed by a single experienced radiology technologist. The scan protocol consisted of a series of standard knee sequences: proton-density weighted (PDW) frequency selective fat suppressed axial (TR/TE 1900/18 ms, TSE factor 6, FOV $150 \times 150 \times 115$, matrix $288 \times 228$, slice thickness $3 \mathrm{~mm}$ ), PDW DRIVE sagittal and coronal (TR/TE 2225/25 ms, TSE factor 12 , FOV $150 \times 150 \times 86$, matrix $432 \times 336$, slice thickness $3 \mathrm{~mm}$ ), and a $3 \mathrm{D}$ gradient echo fat-suppressed sagittal scan. In addition, a sagittal T2-mapping scan (vide infra) was performed.

Controls: Controls from the geMstoan study underwent an MRI using the same 3.0T MRI scanner and eight-channel knee coil as the patients with acromegaly. Both axial and sagittal contrast-enhanced (CE), T1-weighted, turbo spin echo (TSE) and spectral presaturation with inversion recovery (SPIR) sequences were acquired. The control MRI examination did not include a T2-mapping scan.

\section{Study parameters MRI}

Evaluation of structural OA changes on MRI: Knee Osteoarthritis Scoring System (KOSS): MRIs of both patients and controls from the geMstoan study were scored according to the KOSS, which is a validated scoring system for quantifying OA changes in the knee, developed by Kornaat et al. (29). For the present study, cartilaginous defects (diffuse and focal), osteophytes, subchondral cysts and bone marrow edema were graded on a scale of 0 (absent) to 3 (severe). Lesions were localized to any of five regions: medial femoral compartment, medial tibiofemoral compartment, lateral femoral compartment, lateral tibiofemoral compartment and patellofemoral compartment. An osteoarthritic defect was present when a score of $\geq 1$ was given; a severe osteoarthritic defect was defined as KOSS $\geq 2$. MRI scans of patients and controls were scored by an experienced musculoskeletal radiologist (A N C), blinded for any patient characteristics and according to the same protocol. Reproducibility seemed to be high, as assessed by repeated scoring of a small sample of three MRI scans by the same observer in the absence of information of the initial assessment.

Cartilage thickness measurements: In patients and controls form the geMstoan study, cartilage thickness (in 


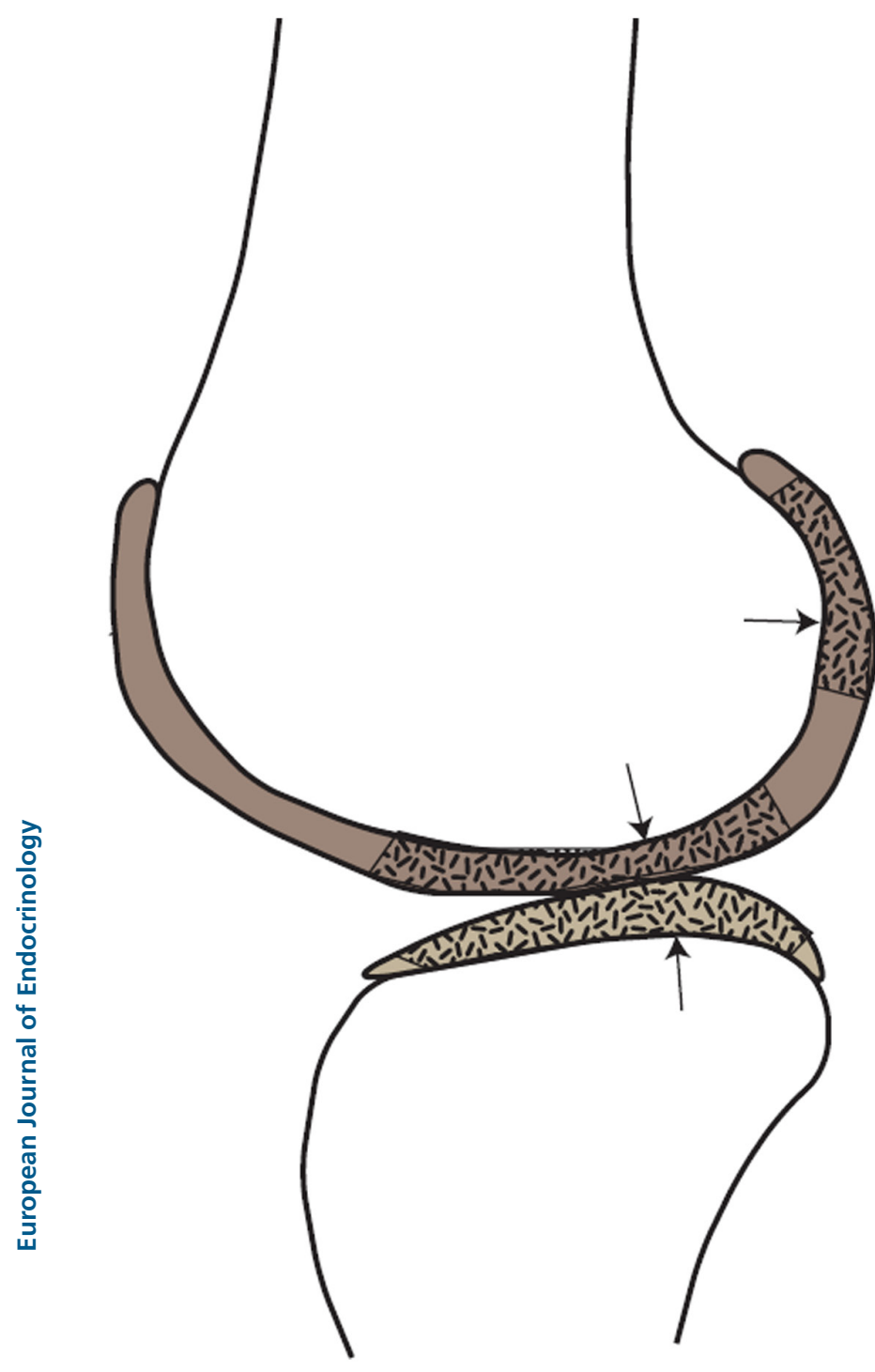

Figure 1

Schematic representation of the ROIs drawn in the medial and lateral condyles of the knee. The arrows indicate the approximate locations of the knee where the ROls were drawn. A full color version of this figure is available at http:// dx.doi.org/10.1530/EJE-16-1073.

millimeters, $\mathrm{mm}$ ) was measured by the same experienced reader $(\mathrm{A} \mathrm{N} \mathrm{C})$ at the level of the tibial attachment of the anterior cruciate ligament (ACL) and in the area with maximum thickness at the lateral and medial femorotibial compartment on the PDW DRIVE coronal weighted images. Cartilage thickness was also measured at the lateral and medial patellofemoral compartments on the PD with fat suppression axial weighted images. Cartilage thickness was not normally distributed with a range in patients of $0.8-4.7 \mathrm{~mm}$ at femoral level and $1.2-5.9 \mathrm{~mm}$ at tibial level; in controls of $1.2-3.1 \mathrm{~mm}$ at femoral level and $1.1-4.4 \mathrm{~mm}$ at tibial level. Reproducibility was good as reflected by an ICC of 0.82 and was based on a random selection of 3 knee MRIs.

\section{T2 mapping}

T2 mapping in patients with acromegaly was performed using a sagittal 2D turbo spin-echo sequence with TR $3307 \mathrm{~ms}, 7$ echoes with TE1/DTE/TE7 13/13/91 ms, acquisition matrix $480 \times 300$, in-plane resolution of $0.31 \times 0.5 \mathrm{~mm}^{2}$, slice thickness $3 \mathrm{~mm}$, FOV $150 \mathrm{~mm}$, and acquisition time $7 \mathrm{~min} 7 \mathrm{~s}$. T2 maps were fitted using the on-scanner vendor-provided software based on a maximum likelihood approach.

ROIs were drawn manually on sagittal slices approximately through the center of the medial and lateral condyles in three locations: the weight-bearing and non-weight-bearing femoral cartilage, and the cartilage of the tibia plateau (Fig. 1). Obvious defects in the cartilage were excluded from the ROIs. The analysis was performed with an algorithm developed in Matlab (MathWorks, Natick, Massachusetts, USA). In 23 patients with acromegaly, T2 maps of sufficient quality (motionand artifact-free) were available, and were hence included in the present analysis. Cartilage relaxation times were measured in milliseconds (ms) with a range of $17.6-45.5 \mathrm{~ms}$ at tibial level and a range of 30.3 $66.0 \mathrm{~ms}$ at femoral level in patients with acromegaly, indicating a relative high variability between patients. Reproducibility of cartilage $\mathrm{T} 2$ relaxation times was moderate, as reflected by an ICC of 0.530 , based on repeated segmentation of random selection of 5 knee MRIs throughout the scoring process, blinded for any patient characteristics.

\section{Statistical analysis}

SPSS for Windows, version 20.0 (SPSS), was used for data analysis. Data are presented as mean \pm S.D., unless otherwise stated. $P$ value was statistically significant at $P<0.05$. The difference in prevalence of structural abnormalities was assessed between active and controlled patients with acromegaly, using a logistic regression model, adjusting for age, sex and BMI. The relationship between different acromegaly-specific parameters (as independent variables) and structural abnormalities (as dependent variables) was also assessed in a logistic regression model. The difference 
Table 1 Clinical characteristics of patients with active acromegaly, patients in remission of acromegaly and controls with primary OA. Data are reported as mean \pm s.D., unless stated otherwise. Control subjects were diagnosed with primary knee OA, and were derived from the geMstoan study (21).

\begin{tabular}{l}
\hline \\
\hline Age (year) \\
Female sex $(n(\%))$ \\
BMI $\left(\mathrm{kg} / \mathrm{m}^{2}\right)$ \\
Active disease duration (year) \\
GH levels (U/L) \\
At diagnosis \\
Current \\
IGF-1 SDS \\
At diagnosis \\
Current \\
Therapy ( $n$ (\%)) \\
Surgery \\
Radiotherapy \\
SMS analogs \\
Pegvisomant \\
D2-agonists \\
Hypopituitarism $(n(\%))$ \\
ACTH deficiency \\
TSH deficiency \\
LH/FSH deficiency \\
ADH deficiency \\
Previous knee surgery $(n(\%)) *$ \\
KL grade $(n(\%)) * *$ \\
Mean KL score \\
Grade 0 \\
Grade 1 \\
Grade 2 \\
Grade 3 \\
Grade 4 \\
Missing X-ray \\
\hline
\end{tabular}

\begin{tabular}{c}
\hline ACRO active disease $(n=10)$ \\
\hline $50.6(13.8)$ \\
$4(40 \%)$ \\
$28.1(2.3)$ \\
$7.8(5.6)$ \\
$163.6(310.7)$ \\
$27.3(24.7)$ \\
$7.4(1.7)$ \\
$5.6(3.6)$ \\
$1(10 \%)$ \\
$0(0 \%)$ \\
$0(0 \%)$ \\
$0(0 \%)$ \\
$0(0 \%)$ \\
$0(0 \%)$ \\
$0(0 \%)$ \\
$2(20 \%)$ \\
$1(10 \%)$ \\
$0(0 \%)$ \\
$1.13(0.84)$ \\
$2(20 \%)$ \\
$3(30 \%)$ \\
$3(30 \%)$ \\
$0(0 \%)$ \\
$0(0 \%)$ \\
$2(20 \%)$ \\
\\
\end{tabular}

\begin{tabular}{c}
\hline ACRO remission $(n=16)$ \\
\hline $60.6(11.9)$ \\
$2(13 \%)$ \\
$30.0(4.8)$ \\
$8.3(6.5)$ \\
$48.0(47.0)$ \\
$3.6(5.3)$ \\
$7.0(4.7)$ \\
$1.0(1.5)$ \\
$13(81 \%)$ \\
$2(13 \%)$ \\
$8(50 \%)$ \\
$4(25 \%)$ \\
$2(13 \%)$ \\
$2(13 \%)$ \\
$1(6 \%)$ \\
$3(19 \%)$ \\
$0(0 \%)$ \\
$2(13 \%)$ \\
\\
$0.94(1.34)$ \\
$9(56 \%)$ \\
$3(19 \%)$ \\
$1(6 \%)$ \\
$2(13 \%)$ \\
$1(6 \%)$ \\
$2(13 \%)$ \\
\\
\\
\\
\\
\\
\end{tabular}

\begin{tabular}{c}
\hline Primary OA $(n=25)$ \\
\hline $59.6(8.0)$ \\
$13(52 \%)$ \\
$28.4(5.3)$ \\
NA \\
NA \\
NA \\
NA \\
\\
\\
NA \\
$3(24 \%)$ \\
$3(12 \%)$ \\
$0(0 \%)$ \\
$2(8 \%)$ \\
$9(36 \%)$ \\
$20(1.08)$ \\
\\
\\
\\
\\
\\
\\
\\
\\
\end{tabular}

*Previous knee surgery of the scanned knee. **KL score of the scanned knee (ACRO, right knee; Controls, knee with symptomatic OA). ACTH, adrenocorticotrophic hormone; ADH, anti-diuretic hormone; BMI, body mass index; D2 agonists, dopamine 2 agonists; FSH, follicle-stimulating hormone; GH, growth hormone; KL, Kellgren-Lawrence score for the presence of radiographic OA; LH, luteinizing hormone; $N$, number of patients; NA, not applicable; OA, osteoarthritis; SMS, somatostatin analogs; TSH, thyroid-stimulating hormone.

in mean cartilage thickness and cartilage $\mathrm{T} 2$ relaxation times was defined between active and controlled patients with acromegaly by a linear regression model, adjusted for age, sex and BMI.

For the comparison of the prevalence of structural OA changes between patients with acromegaly and controls with primary $\mathrm{OA}$, we used a logistic regression model with adjustments for age, sex and BMI. Mean cartilage thickness was compared between patients and controls from the geMstoan study in a linear regression model, adjusting for age, sex and BMI. For comparison of cartilage $\mathrm{T} 2$ relaxation times between patients with acromegaly and 17 controls with mild OA from the literature reference of Stahl et al. (13), a pooled variance $T$ test was performed.

\section{Results}

\section{Characteristics of patients and controls}

Twenty-six patients with acromegaly were included, comprising 10 patients with active acromegaly, of whom 9 patients were treatment-naïve (mean age $50.6 \pm 13.8$ year, $40 \%$ female), and 16 patients in biochemical remission (mean age $60.6 \pm 11.9$ year, $12.5 \%$ female), achieved by transsphenoidal surgery, radiotherapy and/or SMS analogs. Mean remission duration in the latter group was $13.2 \pm 10.6$ year. Two patients, both in remission, had a history of knee arthroscopy in the scanned knee, in the form of a partial meniscectomy in both patients. The mean KL score was comparable between active and controlled patients. 
Table 2 Prevalence of joint defects on a 3.0T MRI of the knee using the KOSS, in patients with active acromegaly $(n=10)$ vs patients with acromegaly in biochemical remission $(n=16)$. Data are presented as $n(\%)$. The difference in prevalence of structural abnormalities between patients with active and controlled acromegaly was assessed using a logistic regression model with structural OA abnormalities as dependent variable and remission state, age, sex and BMI as independent variables. OA defects were scored according to the KOSS score, and were defined as KOSS $\geq 1$.

\begin{tabular}{|c|c|c|c|}
\hline Joint defects & $\begin{array}{c}\text { Active disease } \\
\quad(n=10)\end{array}$ & $\begin{array}{c}\text { Remission } \\
(n=16)\end{array}$ & $P$ value \\
\hline \multicolumn{4}{|l|}{ Cartilage defects } \\
\hline PF & $5(50 \%)$ & $15(94 \%)$ & 0.012 \\
\hline TF & $7(70 \%)$ & $12(75 \%)$ & NS \\
\hline PF and/or TF & $7(70 \%)$ & $16(100 \%)$ & 0.022 \\
\hline \multicolumn{4}{|l|}{ Osteophytes } \\
\hline PF & $7(70 \%)$ & $11(69 \%)$ & NS \\
\hline TF & $8(80 \%)$ & $13(81 \%)$ & NS \\
\hline PF and/or TF & $8(80 \%)$ & $13(81 \%)$ & NS \\
\hline \multicolumn{4}{|c|}{ Subchondral cysts } \\
\hline PF & $0(0 \%)$ & $0(0 \%)$ & NS \\
\hline TF & $0(0 \%)$ & $2(13 \%)$ & NS \\
\hline $\mathrm{PF}$ and/or TF & $0(0 \%)$ & $2(13 \%)$ & NS \\
\hline \multicolumn{4}{|l|}{ BM lesions } \\
\hline PF & $1(10 \%)$ & $6(38 \%)$ & NS \\
\hline $\mathrm{TF}$ & $3(30 \%)$ & $6(38 \%)$ & NS \\
\hline $\mathrm{PF}$ and/or TF & $3(30 \%)$ & $8(50 \%)$ & NS \\
\hline
\end{tabular}

BM, bone marrow; KOSS, Knee Osteoarthritis Scoring System; NS, non-significant; PF, patellofemoral; TF, tibiofemoral.

Patients were compared with 25 controls from the geMstoan study diagnosed with primary OA (mean age $59.6 \pm 8.0$ year, $52 \%$ female). Clinical characteristics of patients and controls were shown in Table 1. Mean age and BMI were comparable between patients and controls, but the control group comprises more females $(P=0.034)$. Definite radiographic knee OA of the scanned knee, defined as $\mathrm{KL} \geq 2$, was present in 7 (27\%) patients and 20 (80\%) controls.

\section{Patients with acromegaly: active vs controlled acromegaly}

\section{Structural abnormalities in the knee according to} the KOSS

Structural abnormalities were already present in a high proportion of patients with active acromegaly. Prevalence of osteophytes, bone marrow lesions and subchondral cysts was comparably high in active and controlled patients with acromegaly. Prevalence of cartilage defects seemed to be higher after achievement of disease remission, although it has to be mentioned that controlled patients tended to be older than active patients $(P=0.061)$ (Table 2$)$.

\section{Cartilage morphometry}

As shown in Table 3, mean total cartilage thickness (i.e. sum of all measured sites) was 8\% higher in patients with active acromegaly than in patients with controlled disease. This was significant, also after adjustments for age, sex and BMI $(P=0.029)$. Especially at the femorotibial compartment, knee cartilage was thicker in patients with active acromegaly than in controlled patients (6\% higher).

In the total acromegaly group, total cartilage thickness correlated to the current IGF-1 SDS value $(r=0.452, P=0.023)$, but not to the pre-treatment IGF-1 SDS values. Cartilage

Table 3 Comparison of mean cartilage thickness in the knee between patients with active acromegaly and patients in remission of acromegaly. Data are presented as mean \pm S.D. Mean cartilage thickness measurements were analyzed using a linear regression model with adjustments for age, sex and BMI. Total cartilage thickness was defined as the sum of all measured sites. Cartilage thickness measurements were collected from 25 patients.

\begin{tabular}{|c|c|}
\hline Cartilage thickness (mm) & Active disease $(n=10)$ \\
\hline \multicolumn{2}{|l|}{ Femorotibial compartment } \\
\hline Medial femoral & $3.01(1.11)$ \\
\hline Lateral femoral & $2.56(0.66)$ \\
\hline Medial tibial & $3.73(0.58)$ \\
\hline Lateral tibial & $4.56(1.19)$ \\
\hline Femorotibial compartment & $13.86(2.67)$ \\
\hline \multicolumn{2}{|l|}{ Patellofemoral compartment } \\
\hline Medial & $3.38(0.70)$ \\
\hline Lateral & $4.02(1.00)$ \\
\hline Patellofemoral compartment & $7.40(1.39)$ \\
\hline Total cartilage thickness & $21.26(3.59)$ \\
\hline
\end{tabular}

\begin{tabular}{r}
\hline Remission $(n=16)$ \\
\hline $2.78(1.15)$ \\
$2.46(0.43)$ \\
$3.69(0.67)$ \\
$4.13(0.98)$ \\
$13.06(2.36)$ \\
$3.21(1.37)$ \\
$3.46(1.53)$ \\
$6.67(2.47)$ \\
$19.73(3.91)$
\end{tabular}

Adjusted mean difference ${ }^{\dagger}(95 \% \mathrm{Cl}) \overline{\text { Adjusted } \boldsymbol{P} \text { value* }}$

${ }^{*}$ Adjusted for age, sex and BMI. ${ }^{\dagger}$ Data presented as $\beta(95 \% \mathrm{Cl})$. NS, non-significant. 
Table 4 Comparison of cartilage T2 relaxation times between patients with active acromegaly and patients in biochemical remission. Data are presented as mean \pm s.D. Cartilage $\mathrm{T} 2$ relaxation times were compared between patients with active and controlled acromegaly using a linear regression model with adjustments for age, sex and BMI.

\begin{tabular}{l}
\hline T2 relaxation times $(\mathrm{ms})$ \\
\hline Femur \\
Medial femoral condyle $(\mathrm{wb})$ \\
Medial femoral condyle $(\mathrm{nwb})$ \\
Lateral femoral condyle $(\mathrm{wb})$ \\
Lateral femoral condyle (nwb) \\
Tibia \\
Medial plateau \\
Lateral plateau
\end{tabular}

\begin{tabular}{c}
\hline Active disease $(n=9)$ \\
\hline \\
$39.6 \pm 4.3$ \\
$48.4 \pm 5.6$ \\
$33.3 \pm 4.1$ \\
$42.9 \pm 2.9$ \\
$42.7 \pm 7.4$ \\
$31.3 \pm 5.5$ \\
\hline
\end{tabular}

\begin{tabular}{c}
\hline Remission $(n=14)$ \\
\hline $44.0 \pm 8.3$ \\
$45.5 \pm 5.7$ \\
$32.9 \pm 4.7$ \\
$41.9 \pm 4.2$ \\
$39.6 \pm 7.1$ \\
$30.7 \pm 6.8$ \\
\hline
\end{tabular}

\begin{tabular}{c} 
Adjusted mean difference $(95 \% \mathrm{Cl})$ \\
\hline$-1.15(-9.05,6.74)$ \\
$5.25(01.17,10.33)$ \\
$1.11(-3.30,5.52)$ \\
$1.00(-7.35,9.35)$ \\
\\
$0.54(-5.01,6.09)$ \\
$6.51(0.76,12.27)$ \\
\hline
\end{tabular}

Adjusted $P$ value*

*Adjusted for age, sex and BMI.

ms, milliseconds; NS, non-significant; nwb, non-weight-bearing; wb, weight-bearing.

thickness was not associated to the presence of structural abnormalities according to the KOSS (data not shown).

\section{Biochemical cartilage composition: cartilage T2 relaxation times}

On all sites, except for the weight-bearing medial femoral condyle, T2 relaxation times were higher in patients with active acromegaly, which reached statistical significance in the non-weight-bearing medial femoral condyle and lateral tibial plateau (Table 4), adjusted for age, sex and BMI. Cartilage T2 relaxation times were not related to

Table 5 Prevalence of joint defects on a 3.0T MRI of the knee using the KOSS, in patients with acromegaly vs controls with primary OA. Data are presented as $\mathrm{n}(\%)$. OA defects were scored according to the KOSS, and were defined as $K O S S \geq 1$. Control subjects were diagnosed with primary knee $\mathrm{OA}$, and were derived from the geMstoan Study (16).

\begin{tabular}{|c|c|c|c|}
\hline Joint defects & $\begin{array}{c}\text { Acromegaly } \\
\quad(n=26)\end{array}$ & $\begin{array}{c}\text { Primary OA } \\
(n=25)\end{array}$ & $P$ value \\
\hline \multicolumn{4}{|l|}{ Cartilage defects } \\
\hline PF & $18(69 \%) *$ & $18(72 \%)$ & \\
\hline TF & $19(73 \%)$ & $20(80 \%)$ & \\
\hline PF and/or TF & $22(85 \%)$ & $23(92 \%)$ & NS \\
\hline \multicolumn{4}{|l|}{ Osteophytes } \\
\hline PF & $17(65 \%)$ & $24(96 \%)$ & \\
\hline TF & $20(77 \%)$ & $22(88 \%)$ & \\
\hline PF and/or TF & $24(92 \%)$ & $24(96 \%)$ & NS \\
\hline \multicolumn{4}{|c|}{ Subchondral cysts } \\
\hline PF & $2(8 \%)$ & $7(28 \%)$ & \\
\hline TF & $1(4 \%)$ & $7(28 \%)$ & \\
\hline PF and/or TF & $3(12 \%)$ & $12(48 \%)$ & 0.001 \\
\hline \multicolumn{4}{|l|}{ BM lesions } \\
\hline PF & $3(12 \%)$ & $13(52 \%)$ & \\
\hline TF & $2(8 \%)$ & $16(64 \%)$ & \\
\hline PF and/or TF & $4(15 \%)$ & $20(80 \%)$ & 0.006 \\
\hline
\end{tabular}

pre-treatment IGF-1 SDS, current IGF-1 SDS or active disease duration (data not shown).

\section{Comparison acromegaly subjects with primary OA}

\section{Structural OA changes according to the KOSS}

As depicted in Table 5, patients with acromegaly have less cysts $(P=0.001)$ and bone marrow lesions $(P=0.006)$ than subjects with primary OA, but comparable prevalence of osteophytosis (although less osteophytosis at the patellofemoral compartment was observed) and cartilage defects (Fig. 2). Prevalence of severe cysts and bone marrow lesions, defined as KOSS $\geq 2$, was comparable between patients with acromegaly and controls with primary OA, whereas the prevalence of severe cartilage

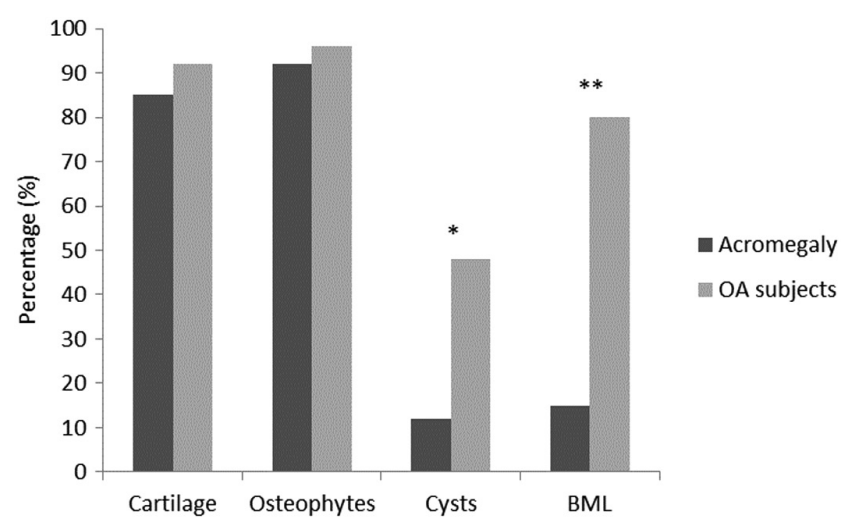

\section{Figure 2}

Structural OA changes detected on MRI in patients with acromegaly vs controls with primary OA. Data were presented as prevalence (\%) of structural joint defects, according to the KOSS. KOSS, Knee Osteoarthritis Scoring System; BML, bone marrow lesions. ${ }^{*} P=0.001 ; * * P=0.006$. 


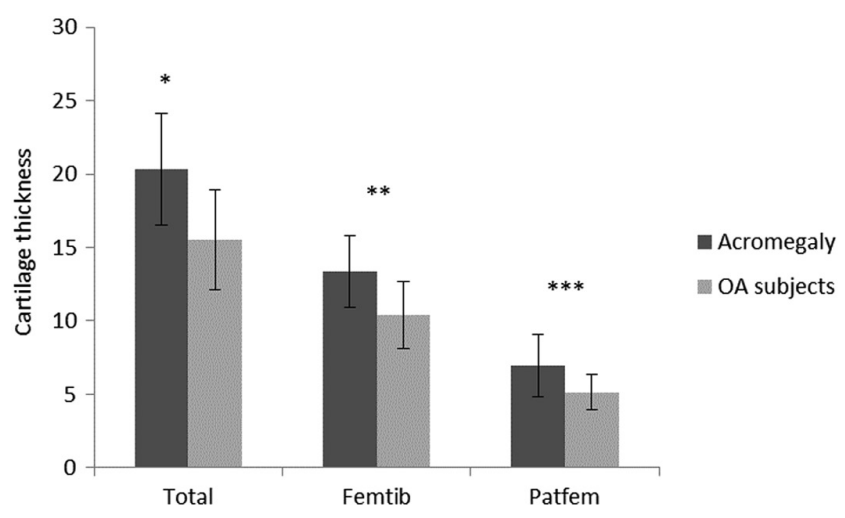

Figure 3

Cartilage thickness in the knee visualized on MRI in patients with acromegaly vs controls with primary OA. Total cartilage thickness measurements were shown as well as the separated values for respectively the femorotibial and patellofemoral compartment. mm, millimeters; Femtib, femorotibial compartment; Patfem, patellofemoral compartment. ${ }^{*} P<0.001 ; * * P=0.002 ; * * * P=0.028$. Analyses were adjusted for age, sex and BMI.

defects and severe osteophytosis was higher in patients with acromegaly.

\section{Cartilage thickness}

Cartilage thickness was significantly higher in patients with acromegaly than in subjects with primary OA, at almost all measured locations at knee (Fig. 3 and Table 6), with a 31\% higher total cartilage thickness in patients with acromegaly. All patients with acromegaly, except for two patients, had total cartilage thickness values above the mean value that was observed in controls.

\section{Biochemical cartilage composition: $T 2$ relaxation times}

Cartilage T2 relaxation times were compared between patients with acromegaly and controls with mild OA from literature to assess the biochemical composition of cartilage. Patients had higher cartilage T2 relaxation times than controls at both the femoral and tibial levels (at all measured sites $P<0.01$; Table 7 ), indicating changes in cartilage quality.

\section{Case series: pre- and postoperative knee MRIs in two patients with acromegaly}

The first patient with acromegaly was a 56-year-old male (patient A) with an estimated active disease duration of 8 years (pre-treatment GH levels and pre-treatment IGF-1 SDS were respectively $45.0 \mu \mathrm{g} / \mathrm{L}$ and $+7.70 \mathrm{SDS})$. The second patient was a 68-year-old male (patient B) with an estimated active disease duration of 15 years (pre-treatment GH levels and pre-treatment IGF-1 SDS were respectively $17.8 \mu \mathrm{g} / \mathrm{L}$ and +7.91 SDS). Both patients underwent MRIs of the knee for two times: the first scan was done during the active, treatment-naïve, phase of acromegaly and the second scan was done at least 6 months after achieving biochemical remission (the MRI scans of patient $A$ and patient $\mathrm{B}$ were performed with an interval of 20 months and 10 months).

In patient $\mathrm{B}$, no cartilage abnormalities were observed on MRI in the active acromegaly phase, however, cartilage

Table 6 Comparison of cartilage thickness in the knee between patients with acromegaly and subjects with primary OA. Data are presented as mean \pm S.D. Control subjects were diagnosed with primary knee OA, and were derived from the geMstoan Study (16). Cartilage thickness measurements between patients and controls were compared using a linear regression model with adjustments for age, sex and BMI.

\begin{tabular}{|c|c|c|c|c|}
\hline Cartilage thickness (mm) & Acromegaly patients $(n=26)$ & Primary OA $(n=25)$ & Adjusted mean difference $(95 \% \mathrm{Cl})$ & Adjusted $\boldsymbol{P}$ value* \\
\hline \multicolumn{5}{|l|}{ Femorotibial compartment } \\
\hline Medial femoral & $2.87(1.12)$ & $2.37(0.74)$ & $0.17(-0.34,0.68)$ & NS \\
\hline Lateral femoral & $2.50(0.52)$ & $2.80(0.66)$ & $-0.44(-0.79,-0.09)$ & 0.015 \\
\hline Medial tibial & $3.70(0.62)$ & $2.32(0.86)$ & $1.20(0.77,1.63)$ & $<0.001$ \\
\hline Lateral tibial & $4.29(1.06)$ & $2.90(0.82)$ & $1.10(0.56,1.64)$ & $<0.001$ \\
\hline Femorotibial compartment & $13.34(2.46)$ & $10.39(2.31)$ & $2.03(0.79,3.28)$ & 0.002 \\
\hline \multicolumn{5}{|l|}{ Patellofemoral compartment } \\
\hline Medial & $3.28(1.14)$ & $2.57(0.96)$ & $0.33(-0.16,0.82)$ & NS \\
\hline Lateral & $3.67(1.36)$ & $2.57(1.33)$ & $0.70(0.00,1.39)$ & 0.049 \\
\hline Patellofemoral compartment & $6.95(2.11)$ & $5.14(1.20)$ & $1.03(0.12,1.93)$ & 0.028 \\
\hline Total cartilage thickness & $20.31(3.79)$ & $15.53(3.41)$ & $3.06(1.52,4.59)$ & $<0.001$ \\
\hline
\end{tabular}


Table 7 Comparison of mean cartilage T2 relaxation times between patients with acromegaly and literature controls with primary OA. Data are presented as mean \pm s.D. Control subjects have mild radiographic OA (KL 1 or 2 ) and clinical OA according to the clinical ACR criteria, and were derived from Stahl et al. (17).

\begin{tabular}{|c|c|c|c|}
\hline $\begin{array}{l}\text { T2 relaxation } \\
\text { times (ms) }\end{array}$ & $\begin{array}{c}\text { Acromegaly } \\
\text { patients } \\
(n=25)\end{array}$ & $\begin{array}{l}\text { Literature } \\
\text { controls } \\
(n=17)\end{array}$ & $P$ value \\
\hline \multicolumn{4}{|l|}{ Femur } \\
\hline $\begin{array}{c}\text { Medial femoral } \\
\text { condyle (wb) }\end{array}$ & $42.1 \pm 7.0$ & $33.7 \pm 3.8$ & $<0.01$ \\
\hline $\begin{array}{l}\text { Medial femoral } \\
\text { condyle (nwb) }\end{array}$ & $46.9 \pm 6.0$ & $33.8 \pm 3.0$ & $<0.01$ \\
\hline $\begin{array}{l}\text { Lateral femoral } \\
\text { condyle (wb) }\end{array}$ & $42.4 \pm 3.8$ & $31.2 \pm 3.0$ & $<0.01$ \\
\hline $\begin{array}{l}\text { Lateral femoral } \\
\text { condyle (nwb) }\end{array}$ & $40.9 \pm 6.9$ & $33.3 \pm 2.7$ & $<0.01$ \\
\hline \multicolumn{4}{|l|}{ Tibia } \\
\hline Medial plateau & $33.3 \pm 4.3$ & $27.5 \pm 2.8$ & $<0.01$ \\
\hline Lateral plateau & $31.0 \pm 6.0$ & $27.9 \pm 3.1$ & $<0.01$ \\
\hline
\end{tabular}

ms, milliseconds; nwb, non-weight-bearing; wb, weight-bearing.

defects (grade 2 and 3) were developed after achieving remission. In patient $\mathrm{A}$, no cartilage progression was seen. In both patients, other structural OA abnormalities did not change. After the establishment of biochemical remission, cartilage thickness regressed in patient B when compared to the pre-operative phase; in patient $\mathrm{A}$, a slight increase in cartilage thickness was observed. Cartilage T2 relaxation times did not change over the short follow-up period.

\section{Discussion}

The present study is the first to evaluate acromegalic arthropathy using MRI in acromegaly patients both with active and controlled disease. We found that structural OA abnormalities on MRI were already highly prevalent during active disease, especially of osteophytosis, when compared to patients with controlled acromegaly. In addition, articular knee cartilage was thicker and cartilage T2 relaxation times were higher in patients with active acromegaly than in patients with controlled acromegaly, reflecting differences in cartilage quality between these patients. When compared to subjects with primary OA, acromegalic arthropathy seems to be predominantly characterized by alterations in joint cartilage, being thicker cartilage and changes in biochemical composition of the cartilage. In addition, patients with acromegaly showed less cysts and bone marrow lesions, whereas prevalence of cartilage defects and osteophytosis was comparable.
Arthropathy is one of the most invalidating complications in acromegaly, despite biochemical disease control $(1,2)$, significantly impairing QoL. The exact pathogenesis of acromegalic arthropathy is currently unknown, but there are some similarities with that of primary OA. There is evidence that GH/IGF-1 activity is associated with both the onset and progression of acromegalic arthropathy $(3,4,5)$. Interestingly, patients with acromegaly have a characteristic radiographic phenotype with severe osteophytosis with preservation of joint cartilage $(1,11)$. Until now, these characteristics were only observed in radiographic studies and in a few studies using ultrasonography.

The present study shows that structural OA defects are already highly prevalent during the active acromegaly phase. In addition, we found that in patients with active acromegaly articular cartilage is not only thicker than that in the controlled disease phase, but is also from a different biochemical composition, as reflected by higher cartilage $\mathrm{T} 2$ relaxation times. Cartilage $\mathrm{T} 2$ relaxation times are influenced by several factors, such as the orientation of collagen fibers to the main magnetic field, water content, alterations in water proton mobility and the integrity of collagenous structures in the extracellular cartilage matrix $(30,31,32)$. In previous studies, patients with primary OA showed higher T2 relaxation times than the healthy controls $(14,15)$, with a clear correlation between these values and OA severity, indicating increased water content in these patients. The findings of the present study give rise to a new hypothesis that thickened joint cartilage in patients with active acromegaly consists of two different components: a structural component of cartilage hypertrophy, being (partially) irreversible despite longterm biochemical remission $(11,12)$, and a component of edema (reflected by cartilage $\mathrm{T} 2$ relaxation times), which decreases after successful treatment. This may explain why joint cartilage of patients with controlled acromegaly is still thickened compared to that of healthy controls due to persisting cartilage hypertrophy, but is thinner than that in the active phase due to a decrease in water content by successful treatment. This hypothesis is supported by a corresponding decrease in cartilage $\mathrm{T} 2$ relaxation times in the long term after achievement of biochemical control; although it is currently unknown when this decrease occurs in time after remission has been obtained.

When compared to subjects with primary OA, patients with acromegaly have thicker knee cartilage. These results are in keeping with radiographic studies reporting widened joint spaces (11), indicating persistent (protective) effects of previous GH excess on joint 
cartilage. This may explain why, despite significant joint complaints, joint replacement surgery is less frequently performed in patients with acromegaly, and, in addition, why fewer acromegalics have definite $\mathrm{OA}$ according to the Kellgren and Lawrence Score, which is a JSN-based scoring system. A new finding is the presence of higher cartilage T2 relaxation times in patients with acromegaly at all measured sites, suggesting that average biochemical composition of joint cartilage is altered in these patients. The observation of even higher cartilage $\mathrm{T} 2$ relaxation times in patients with acromegaly might reflect increased cartilage damage in acromegalics, with more cartilage hydration and collagen breakdown. Observations of these altered cartilage composition should be confirmed in future studies.

This study may suffer from several limitations. First, due to the relatively small number of patients, only explorative analyses are carried out in this pilot study. Studies with a larger number of patients are needed to enable more definite conclusions. Secondly, the acromegaly group included in the study is quite heterogeneous, including patients with both active and controlled acromegaly. However, we decided to include treated patients next to treatment-naïve patients, to assess the effects of adequate treatment of acromegaly at the joint level. Thirdly, in the absence of $\mathrm{T} 2$ relaxation time control data in our center, for comparison we were restricted to the inclusion of a literature reference with subjects with primary OA. In this regard, differences between MRI scanners, scan protocols and scoring methods may confound these results. Finally, the cartilage thickness and T2 relaxation measurements are a first exploration of the MRI data, although we believe it is a quite innovative way to study joint cartilage in patients with acromegaly. In future work, we aim to analyze the data in more depth, including thickness measurements over the entire cartilage surface and T2-value assessment in the different cartilage layers. For future scans, these analyses may benefit from the higher resolution, both in the spatial and in the contrast domain, which can be achieved using the 7.0T MRI scanner of the C.J. Gorter Center in our hospital. In addition, the moderate ICC of the T2 mapping might be improved by the implementation of a (semi-)automatic analysis algorithm.

In conclusion, this first MRI study on acromegalic arthropathy demonstrates that in the active acromegaly phase, structural OA defects are already highly prevalent. Patients with active disease have thicker joint cartilage with higher water content than that in patients with controlled disease, as reflected by increased cartilage $\mathrm{T} 2$ relaxation times. At present, it is still unknown when these cartilage changes occur in time after achieving remission. When compared to subjects with primary OA, acromegalic arthropathy especially differs with respect to joint cartilage, which is thicker and from different biochemical composition. The findings of the present study underline that acromegalic arthropathy is a clinical entity with a unique phenotype. Future studies are required to point out whether acromegaly-specific interventions can be beneficial in the management of acromegalic arthropathy.

\section{Supplementary data}

This is linked to the online version of the paper at http://dx.doi.org/10.1530/ EJE-16-1073.

\section{Declaration of interest}

The authors declare that there is no conflict of interest that could be perceived as prejudicing the impartiality of this study.

\section{Funding}

We acknowledge the investigator-initiated research grant provided by Pfizer BV, the Netherlands.

\section{Acknowledgements}

The authors would like to thank A W Visser and B J E de Lange-Brokaar for their assistance with the data collection of the geMstoan Study and their help in processing the MRI scores. H M Kroon and N R Biermasz are shared last authors.

\section{References}

1 Wassenaar MJ, Biermasz NR, van Duinen N, van der Klaauw AA, Pereira AM, Roelfsema F, Smit JW, Kroon HM, Kloppenburg M $\&$ Romijn JA. High prevalence of arthropathy, according to the definitions of radiological and clinical osteoarthritis, in patients with long-term cure of acromegaly: a case-control study. European Journal of Endocrinology 2009160 357-365. (doi:10.1530/EJE-08-0845)

2 Wassenaar MJ, Biermasz NR, Kloppenburg M, van der Klaauw AA, Tiemensma J, Smit JW, Pereira AM, Roelfsema F, Kroon HM \& Romijn JA. Clinical osteoarthritis predicts physical and psychological QoL in acromegaly patients. Growth Hormone and IGF Research 201020 226-233. (doi:10.1016/j.ghir.2010.02.003)

3 Biermasz NR, Wassenaar MJ, van der Klaauw AA, Pereira AM, Smit JW, Roelfsema F, Wolterbeek R, Kroon HM, Kloppenburg M \& Romijn JA. Pretreatment insulin-like growth factor-I concentrations predict radiographic osteoarthritis in acromegalic patients with long-term cured disease. Journal of Clinical Endocrinology and Metabolism 200994 2374-2379. (doi:10.1210/jc.2008-2393)

4 Claessen KM, Ramautar SR, Pereira AM, Smit JW, Roelfsema F, Romijn JA, Kroon HM, Kloppenburg M \& Biermasz NR. Progression of acromegalic arthropathy despite long-term biochemical control: a prospective, radiological study. European Journal of Endocrinology 2012 167 235-244. (doi:10.1530/EJE-12-0147)

5 Wassenaar MJ, Biermasz NR, Pereira AM, van der Klaauw AA, Smit JW, Roelfsema F, van der Straaten T, Cazemier M, Hommes DW, 
Kroon HM et al. The exon-3 deleted growth hormone receptor polymorphism predisposes to long-term complications of acromegaly. Journal of Clinical Endocrinology and Metabolism 200994 4671-4678. (doi:10.1210/jc.2009-1172)

6 Colao A, Marzullo P, Vallone G, Marinò V, Annecchino M, Ferone D, De Brasi D, Scarpa R, Oriente P \& Lombardi G. Reversibility of joint thickening in acromegalic patients: an ultrasonography study. Journal of Clinical Endocrinology and Metabolism 199883 212-215. (doi:10.1210/jcem.83.6.4865)

7 Colao A, Ferone D, Marzullo P \& Lombardi G. Systemic complications of acromegaly: epidemiology, pathogenesis, and management. Endocrine Reviews 200425 102-152. (doi:10.1210/er.2002-0022)

8 Lieberman SA, Bjorkengren AG \& Hoffman AR. Rheumatologic and skeletal changes in acromegaly. Endocrinology Metabolism Clinics of North America 199221 615-631.

9 Claessen KM, Mazziotti G, Biermasz NR \& Giustina A. Bone and joint disorders in acromegaly. Neuroendocrinology 2016103 86-95. (doi:10.1159/000375450)

10 Barkan AL. Acromegalic arthropathy. Pituitary 20014 263-264. (doi:10.1023/A:1020754615863)

11 Wassenaar MJ, Biermasz NR, Bijsterbosch J, Pereira AM, Meulenbelt I, Smit JW, Roelfsema F, Kroon HM, Romijn JA \& Kloppenburg M. Arthropathy in long-term cured acromegaly is characterised by osteophytes without joint space narrowing: a comparison with generalised osteoarthritis. Annals of the Rheumatic Diseases 201170 320-325. (doi:10.1136/ard.2010.131698)

12 Colao A, Cannavo S, Marzullo P, Pivonello R, Squadrito S, Vallone G, Almoto B, Bichisao E, Trimarchi F \& Lombardi G. Twelve months of treatment with octreotide-LAR reduces joint thickness in acromegaly. European Journal of Endocrinology 2003148 31-38. (doi:10.1530/ eje.0.1480031)

13 Stahl R, Luke A, Li X, Carballido-Gamio J, Ma CB, Majumdar S \& Link TM. T1rho, T2 and focal knee cartilage abnormalities in physically active and sedentary healthy subjects versus early OA patients - a 3.0Tesla MRI study. European Radiology 200919 132-143. (doi:10.1007/ s00330-008-1107-6)

14 Blumenkrantz G, Lindsey CT, Dunn TC, Jin H, Ries MD, Link TM, Steinbach LS \& Majumdar S. A pilot, two-year longitudinal study of the interrelationship between trabecular bone and articular cartilage in the osteoarthritic knee. Osteoarthritis and Cartilage 200412 997-1005. (doi:10.1016/j.joca.2004.09.001)

15 Dunn TC, Lu Y, Jin H, Ries MD \& Majumdar S. T2 relaxation time of cartilage at MR imaging: comparison with severity of knee osteoarthritis. Radiology 2004232 592-598. (doi:10.1148/ radiol.2322030976)

16 de Lange-Brokaar BJE, Ioan-Facsinay A, Yusuf E, Visser AW, Kroon HM, Andersen SN, Herb-van Toorn L, van Osch GJ, Zuurmond AM, Stojanovic-Susulic V et al. Degree of synovitis on MRI is by comprehensive whole knee semi-quantitative scoring method correlates with histologic and macroscopic features of synovial tissue inflammation in knee osteoarthritis. Osteoarthritis and Cartilage 2014 22 1606-1613. (doi:10.1016/j.joca.2013.12.013)

17 Biermasz NR, van Dulken H \& Roelfsema F. Ten-year follow-up results of transsphenoidal microsurgery in acromegaly. Journal of Clinical Endocrinology and Metabolism 200085 4596-4602. (doi:10.1210/ jcem.85.12.7042)

18 Biermasz NR, Pereira AM, Smit JW, Romijn JA \& Roelfsema F. Morbidity after long-term remission for acromegaly: persisting jointrelated complaints cause reduced quality of life. Journal of Clinical
Endocrinology and Metabolism 200590 2731-2739. (doi:10.1210/ jc.2004-2297)

19 Giustina A, Chanson P, Bronstein MD, Klibanski A, Lamberts S, Casanueva FF, Trainer P, Ghigo E, Ho K, Melmed S et al. A consensus on criteria for cure of acromegaly. Journal of Clinical Endocrinology and Metabolism 201095 3141-3148. (doi:10.1210/jc.2009-2670)

20 Trainer PJ, Drake WM, Katznelson L, Freda PU, Herman-Bonert V, van der Lely AJ, Dimaraki EV, Stewart PM, Friend KE, Vance ML et al. Treatment of acromegaly with the growth hormone-receptor antagonist pegvisomant. New England Journal of Medicine 2000342 1171-1177. (doi:10.1056/NEJM200004203421604)

21 Fleseriu M, Hashim IA, Karavitaki N, Melmed S, Murad MH, Salvatori $\mathrm{R} \&$ Samuels MH. Hormonal replacement in hypopituitarism in adults: an Endocrine Society Clinical Practice Guideline. Journal of Clinical Endocrinology and Metabolism 2016101 3888-3921. (doi:10.1210/jc.2016-2118)

22 Cole TJ. The LMS method for constructing normalized growth standards. European Journal of Clinical Nutrition $1990 \mathbf{4 4} 45-60$.

23 Rikken B, van DJ, Ringeling A, Van den Brande JL, Massa G $\&$ Wit JM. Plasma levels of insulin-like growth factor (IGF)-I, IGF-II and IGF-binding protein-3 in the evaluation of childhood growth hormone deficiency. Hormone Research 199850 166-176. (doi:10.1159/000023268)

24 Peterfy C, Li J, Zaim S, Duryea J, Lynch J, Miaux Y, Yu W \& Genant HK. Comparison of fixed-flexion positioning with fluoroscopic semiflexed positioning for quantifying radiographic joint-space width in the knee: test-retest reproducibility. Skeletal Radiology 200332 128-132. (doi:10.1007/s00256-002-0603-z)

25 Kellgren JH \& Lawrence JS. Radiological assessment of osteo-arthrosis. Annals of the Rheumatic Diseases 195716 494-502. (doi:10.1136/ ard.16.4.494)

26 Link TM, Stahl R \& Woertler K. Cartilage imaging: motivation, techniques, current and future significance. European Radiology 2007 17 1135-1146. (doi:10.1007/s00330-006-0453-5)

27 Craig JG, Go L, Biechinger J, Hearshen D, Bouffard JA, Diamond M \& van Holsbeeck MT. Three-tesla imaging of the knee: initial experience. Skeletal Radiology 200534 453-461. (doi:10.1007/s00256-005-0919-6)

28 Ramnath RR. 3T MR imaging of the musculoskeletal system (Part I): considerations, coils, and challenges. Magnetic Resonance Imaging Clinics of North America 200614 27-40. (doi:10.1016/j.mric.2006.01.001)

29 Kornaat PR, Ceulemans RY, Kroon HM, Riyazi N, Kloppenburg M, Carter WO, Woodworth TG \& Bloem JL. MRI assessment of knee osteoarthritis: Knee Osteoarthritis Scoring System (KOSS) - inter-observer and intraobserver reproducibility of a compartment-based scoring system. Skeletal Radiology 200534 95-102. (doi:10.1007/s00256-004-0828-0)

30 Lusse S, Claassen H, Gehrke T, Hassenpflug J, Schünke M, Heller M \& Glüer CC. Evaluation of water content by spatially resolved transverse relaxation times of human articular cartilage. Magnetic Resonance Imaging 200018 423-430. (doi:10.1016/S0730725X(99)00144-7)

31 Mosher TJ, Dardzinski BJ \& Smith MB. Human articular cartilage: influence of aging and early symptomatic degeneration on the spatial variation of T2 - preliminary findings at 3 T. Radiology 2000214 259-266. (doi:10.1148/radiology.214.1.r00ja15259)

32 Nissi MJ, Rieppo J, Toyras J, Laasanen MS, Kiviranta I, Jurvelin JS \& Nieminen MT. T(2) relaxation time mapping reveals age- and speciesrelated diversity of collagen network architecture in articular cartilage. Osteoarthritis and Cartilage 200614 1265-1271. (doi:10.1016/j. joca.2006.06.002)
Received 28 December 2016

Revised version received 14 March 2017

Accepted 24 March 2017 